\title{
Deep tissue imaging by enhanced photon collection
}

\author{
Viera Crosignani, Sohail Jahid, Alexander Dvornikov and Enrico Gratton* \\ Department of Biomedical Engineering \\ University of California at Irvine \\ Irvine, CA 92697-2715, USA \\ *egratton@uci.edu
}

Received 18 June 2013

Accepted 16 September 2013

Published 4 June 2014

\begin{abstract}
We have developed a two-photon fluorescence microscope capable of imaging up to $4 \mathrm{~mm}$ in turbid media with micron resolution. The key feature of this instrument is the innovative detector, capable of collecting emission photons from a wider surface area of the sample than detectors in traditional two-photon microscopes. This detection scheme is extremely efficient in the collection of emitted photons scattered by turbid media which allows eight fold increase in the imaging depth when compared with conventional two-photon microscopes. Furthermore, this system also has in-depth fluorescence lifetime imaging microscopy (FLIM) imaging capability which increases image contrast. The detection scheme captures emission light in a transmission configuration, making it extremely efficient for the detection of second harmonic generation (SHG) signals, which is generally forward propagating. Here we present imaging experiments of tissue phantoms and in vivo and ex vivo biological tissue performed with this microscope.
\end{abstract}

Keywords: Deep tissue; microscopy; fluorescence; SHG; FLIM.

\section{Introduction}

Biology and medical diagnostics often require imaging of deep layers of biological tissue with cellular resolution and without disrupting the physiological condition of the sample. Deep-tissue imaging can be regarded as noninvasive "optical pathology", where all cells and molecules are observed in their intact physiological environment.

The ability to image in depth is dependent on both the geometrical and optical properties of the sample, as well as the capabilities of the imaging system. The most common imaging systems available like confocal microscopes are only suitable for relatively transparent samples and cannot be used to access deep layer of biological tissue, which is generally strongly scattering. Tissues are characterized by strong multiple scattering and by inhomogeneity of the refractive index. Traditional light and fluorescence microscopy, even with the aid of staining and using fluorescent markers to improve contrast, cannot image beyond $100-200 \mu \mathrm{m}$ from the surface layer of the turbid sample. ${ }^{1}$

To access deeper layer of turbid samples, high power, ultrashort pulsed lasers in the near infrared

This is an Open Access article published by World Scientific Publishing Company. It is distributed under the terms of the Creative Commons Attribution 3.0 (CC-BY) License. Further distribution of this work is permitted, provided the original work is properly cited. 
(NIR) are required, since they exploit the reduced absorption and scattering at longer wavelengths and use multiphoton absorption to excite molecules in the sample. In spite of the noticeable improvement in imaging depth obtained with multi-photon microscopy, as compared to the conventional confocal microscopy, the state-of-the-art microscope technology still cannot overcome the imaging limit of about $1 \mathrm{~mm} .^{2-5}$

We have built a two-photon fluorescence microscope named the DIVER, which stands for deep imaging via enhanced-photon recovery. The DIVER is capable of imaging turbid samples with brain-like optical properties up to the depth of $4 \mathrm{~mm}$. The large imaging depth is achieved with an innovative photon recovery system which allows the collection of emitted photons from a wide area of the sample, while conventional detection schemes collect photons only from a relatively small area, and thus losing most fluorescence photons. We have also reduced light loss matching the refractive index throughout the optical path between the sample and the detector. We have successfully validated the DIVER using many biological and artificial specimens. As for the artificial samples we have used phantoms simulating brain optical properties. As biological samples we have used excised tissue from mice such as colon and small intestine, vasculature in the skin, and subcutaneous xenograft tumors.

The system operates in transmission geometry, which makes it extremely suitable for second harmonic generation (SHG) signal detection. SHG is a coherent process in which photons are generated at exactly half the wavelength of the excitation light. SHG is a powerful asset in biological and medical imaging and diagnostics. ${ }^{6}$ SHG does not generate contrast by means of absorption, like in the case of multi-photon fluorescence microscopy, but draws on the polarizability of specific endogenous structures lacking center of symmetry, and thus staining of the sample is not necessary. SHG also works at near IR laser wavelengths, which allows imaging deeper in biological tissue with cellular resolution. While fluorescence results in isotropic emission, SHG mainly propagates in the direction of the excitation beam, ${ }^{7}$ which makes SHG detection better accomplished in an imaging system working in transmission geometry.

Due to its distinctive properties, SHG is a very useful imaging technique; it is employed in both ex vivo and in vivo, in the imaging of diseases of connective tissue. ${ }^{8}$ Collagen, a very strong SHG signal generator, is extremely abundant in the extra-cellular matrix (ECM) of tissue. The ability to study the ECM is important in cancer research as it has been shown that ECM remodeling often takes place in many diseases, such as ovarian ${ }^{9}$ and breast cancer. ${ }^{10}$ SHG is also used in studying muscle diseases where myosin is present in large amounts and can be detected with SHG. ${ }^{11}$

The DIVER not only can acquire better in-depth SHG images of biological and nonbiological samples compared to traditional microscope geometry, but it also requires very low excitation light power, and thus limiting photodamage, when compared to traditional methods.

The DIVER is also equipped with a FLIMBox (ISS, Inc., Champaign, IL) for in-depth fluorescence lifetime imaging microscopy (FLIM). FLIM is an imaging contrast method based on the mapping of the spatial distribution of the unique lifetimes of intrinsic and extrinsic fluorophores, such as NADH, FAD, and fluorescent proteins. ${ }^{12}$ FLIM can be used to analyze protein-protein interactions and also in detecting various biological conditions. ${ }^{13,14}$ FLIM analysis performed in the time domain is very computationally demanding, but the introduction of the phasor-approach ${ }^{15}$ by Digman et al., has allowed performing FLIM data analysis in the frequency domain and it has significantly reduced the data processing time.

\section{Experimental Methods}

\subsection{Imaging system}

The imaging system consists of a custom made upright two-photon excitation microscope equipped with a novel emission detection setup. The principles of operation were previously described. ${ }^{16,17}$ This paper describes an updated system, shown schematically in Fig. 1.

Two-photon fluorescence excitation and SHG are provided by tunable femtosecond pulsed Ti:Sa laser (Mai Tai, Spectra Physics, Irvine, CA) coupled to a group velocity dispersion compensator (DeepSee, Spectra Physics, Irvine, CA) to maximize fluorescence excitation efficiency at the sample. The power level of the excitation beam is regulated by an acousto-optic modulator (AOM MT 110-B50A1, AA Opto-Electronics, Orsay, France). The average excitation beam power required for imaging depends 


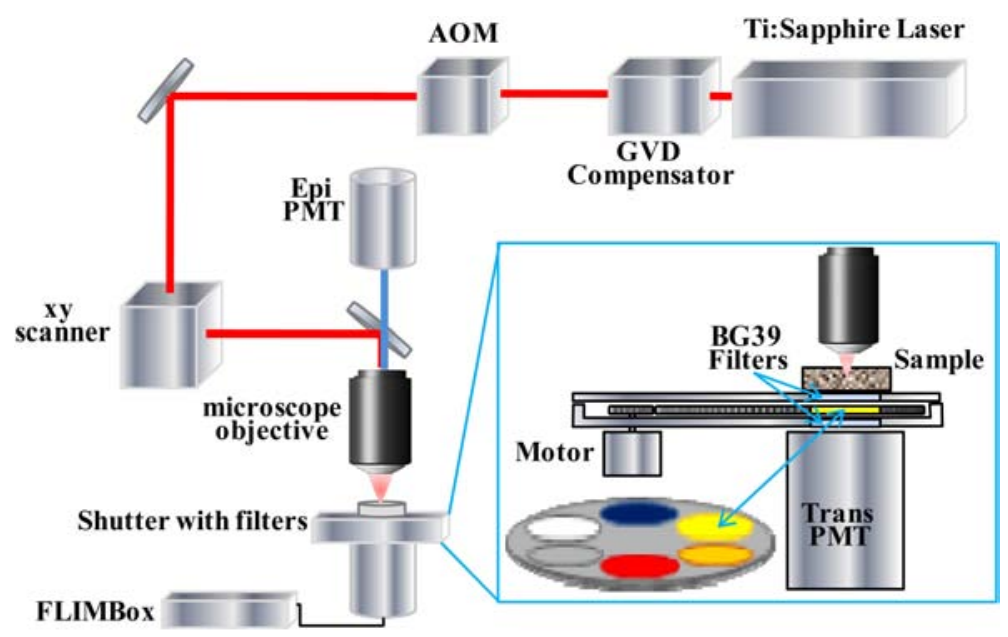

Fig. 1. Schematic diagram of the experimental system.

on the type of sample, fluorophore, and imaging depth and increases with imaging depth usually from few tens of $\mathrm{mW}$ for layers close to the sample surface to few hundreds of $\mathrm{mW}$ for layers at mm depths. We first perform a quick z-scan to measure the attenuation as a function of z. During the scan, we change the laser power to maintain approximately the same detected intensity as we go deep in the sample. Use of high excitation power potentially may cause the sample damage, however, when the excitation beam is focused deep inside the turbid media, for the samples having low absorption at NIR excitation wavelength, which is typical for most biological tissues, these high power levels usually do not induce sample damage due to spread of light energy across the sample by strong multiple scattering. The excitation beam is subsequently directed to a $\mathrm{x}-\mathrm{y}$ galvanometric scanner (ISS, Inc.) coupled with an Olympus BX illumination module equipped with long working distance objectives (LCPlanFl $20 \mathrm{x} / 0.4, \mathrm{WD}=7 \mathrm{~mm} ;$ LUMPlanFl $40 \mathrm{x} / 0.80 \mathrm{~W}$, $\mathrm{WD}=3.3 \mathrm{~mm}, \quad$ XLSLPLN25XSVMP $\quad 25 \mathrm{x} / 0.9$, $\mathrm{WD}=8 \mathrm{~mm}$, Olympus, Tokyo, Japan). The sample is positioned directly on the stage coupled to the detection system (described below) and attached to a motorized $\mathrm{x}-\mathrm{y}-\mathrm{z}$ stage used to position the sample and to focus the laser beam.

The detector, operating in transmission setup, is the core element of the DIVER which has allowed us to extend the imaging depth in turbid samples to $4 \mathrm{~mm} .{ }^{16,17}$ As described above, this system is also particularly effective in the detection of SHG photons, due to their forward-propagating nature. The detector described in this paper has been upgraded by the addition of a filter wheel to separate emission photons by wavelength. The detector consists of a large photocathode area head-on photomultiplier tube (PMT; R7600P-300, Hamamatsu, Japan), working in photon counting mode, coupled to the shutter. The shutter is a custom-made sealed aluminum case equipped with two optical windows made of $25 \mathrm{~mm}$ diameter $\times 3 \mathrm{~mm}$ thick BG-39 filters, Fig. 1. The PMT is attached to the bottom window of the shutter using microscope objective oil to eliminate the air gap and match the index of refraction between the PMT window and the filter. The sample is placed directly on the top shutter window. BG-39 filters prevent the NIR excitation beam from entering the PMT, while transmit visible fluorescence light induced in the sample. The shutter contains the filter wheel rotated by a stepper motor. This filter wheel has 6 positions, corresponding to closed (no light penetrate), open (no filter inserted), blue-violet bandpass B-370 filter and 3 longpass glass filters with cutoff wavelengths of 455,500 , and $550 \mathrm{~nm}$, respectively. These filters are used to separate emission wavelengths. Because the scattered emission light enters the shutter assembly from various directions, we cannot use interference filters that have more precise wavelength selection, but require collimated beam, however, the used glass filters were found to be very useful for imaging. The filter case is filled with index matching fluid to maintain refractive index continuity throughout the optical path between the sample and the PMT window in order to minimize losses of emitted photons caused by multiple reflections at the boundaries. Since the emission photons are 
collected directly from the wide surface area of the sample and, due to refraction index matching, at almost any entering direction, the effective numerical aperture of this detection system is above 1, which makes it extremely sensitive to very low signal levels. The system is also equipped with a FLIMBox (ISS, Inc., Champaign, IL) for in-depth FLIM. A second PMT (H7422P-40, Hamamtsu, Japan) that works in the epi-fluorescence configuration has also been added to the system as a comparative module between the DIVER and conventional two-photon fluorescence microscope configuration.

All image acquisitions, 3D reconstructions, and data analysis were performed using the SimFCS software developed at laboratory for fluorescence dynamics (LFD; the commercial version "Globals for Images" is available at www.lfd.uci.edu). The images consisting of $256 \times 256$ pixels were acquired by raster scanning with dwell time of $32 \mu \mathrm{s} /$ pixel that takes approximately $2.5 \mathrm{~s}$ to acquire one frame. Typically 15-20 frames were averaged for each image plane. Because the signal intensity decreases with imaging depth, the excitation power needs to be increased to compensate for this effect. However, at high excitation power levels, the phenomenon known as out-offocus fluorescence may be induced at a sample surface layer, which further decreases the signal-to-noise ratio. In experiments on imaging fluorescent beads sandwiched between two 4-mm thick slices of scattering silicone phantoms, the signal-to-noise ratio was measured to be about 1.7 and 60 frames were accumulated and averaged to produce the image.

\subsection{Sample preparation}

Silicone tissue phantoms (reduced scattering coefficient $\mu_{s}^{\prime}=1.13 \mathrm{~mm}^{-1}$, scattering anisotropy $g=0.9$ at $800 \mathrm{~nm}$ excitation wavelength) were prepared according to Ref. 18 with the addition of yellowgreen $(1,2$ and $15 \mu \mathrm{m})$ and red $(10 \mu \mathrm{m})$ fluorescent beads (Invitrogen, Carlsbad, CA). Samples for SHG experiments were analogously prepared with the addition of urea crystals (Aldrich, St. Louis, MO), which were powdered in a mortar prior to mixing. This phantom sample had dimensions of $35 \mathrm{~mm}$ in diameter and $8 \mathrm{~mm}$ thickness. The preparation of collagen matrix samples was described in Ref. 19. Xenograft tumors were grown subcutaneously in immune-deficient mice and were excised from a skin flap and imaged immediately post-euthanization. The thickness of tissue samples was about $2-3 \mathrm{~mm}$.
Tissue was obtained from 20-week old wild-type mice on approved IACUC protocol 2011-2978-0 (University of California Irvine). Tissues were obtained post-mortem and placed in cold PBS.

\section{Results and Discussion}

\subsection{Images in phantoms}

We have previously ${ }^{16,17}$ shown that the described detection setup allows imaging fluorescent beads in turbid media up to the depth of $3 \mathrm{~mm}$, while the same sample could only be imaged up to the depth of $500 \mu \mathrm{m}$ by a commercial Zeiss LSM 710 microscope. The addition of a more powerful laser and of a group velocity dispersion compensation device to the DIVER has further extended the available imaging depth.

To assess the in-depth imaging capabilities of the DIVER, yellow-green fluorescent beads dispersed in a tissue phantom with brain-like optical properties were imaged. The system has been updated with an automated z-stack feature that allows a 3D reconstruction of the full thickness of a turbid sample. Figure 2(a) shows the 3D reconstruction of a z-stack image of fluorescent beads acquired to $3 \mathrm{~mm}$ depth. The same sample was also imaged with our system in the epi-fluorescence configuration (same objective and excitation parameters) and with the Zeiss LSM 710 and in both cases the $500 \mu \mathrm{m}$ imaging depth limit could not be overcame. When the sample was imaged with the DIVER's wide area detector, even at $3 \mathrm{~mm}$ depth the image maintained high resolution. Next we imaged a cluster of $1 \mu \mathrm{m}$ beads placed between two scattering slices of silicone resin each 4-mm thick [Fig. 2(b)]. Because image intensity decreases with imaging depth in order to compensate for this phenomenon we need to collect more frames at a 4-mm imaging depth to preserve a given image quality. Nevertheless, the beads can be clearly distinguished in the image.

\subsection{Images of biological tissues}

The DIVER was successfully employed in imaging various types of biological tissues. One example is the vasculature of subcutaneous xenograft tumors. Colon cancer cells injected subcutaneously induce a tumor mass that will develop a vasculature network to acquire nourishment. The ability to image and study the vascularization of tumor cells has become 


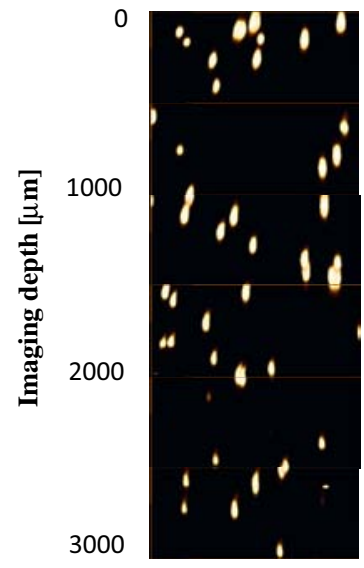

(a)

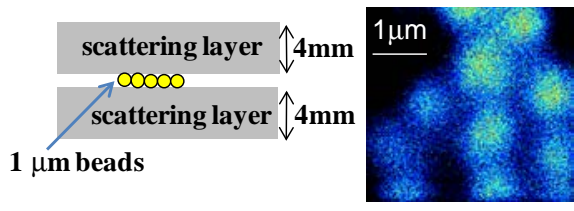

(b)

Fig. 2. 3D image reconstruction of $2 \mu \mathrm{m}$ beads embedded in scattering silicone resin (a). Image of $1 \mu \mathrm{m}$ beads sandwiched between two 4-mm thick scattering silicone slices (b). The Olympus XLSLPLN25XSVMP microscope objective was used to acquire image (b).

important in progression of tumor and so far there have been limitation of in vivo imaging of these vessels. Figure 3 shows the vasculature of the subcutaneous tumor with FITC-Dextran as a contrast agent. This sample was imaged using the DIVER up to depth of $630 \mu \mathrm{m}$.

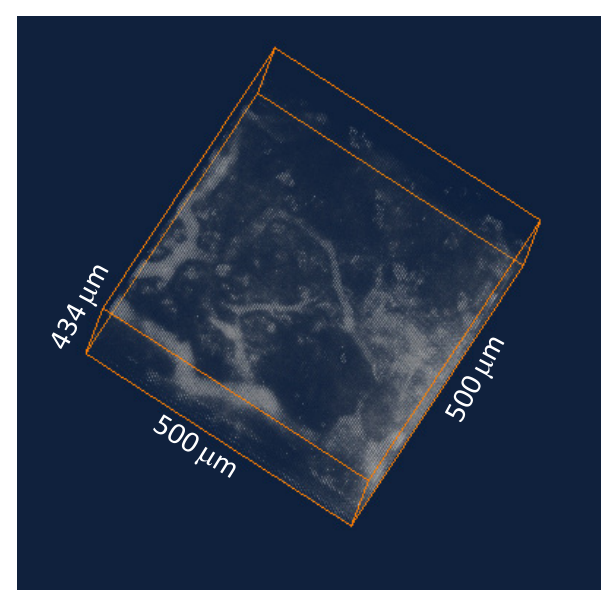

Fig. 3. Vasculature of the subcutaneous tumor with FITCDextran as a contrast agent at the depth of $630 \mu \mathrm{m}$.

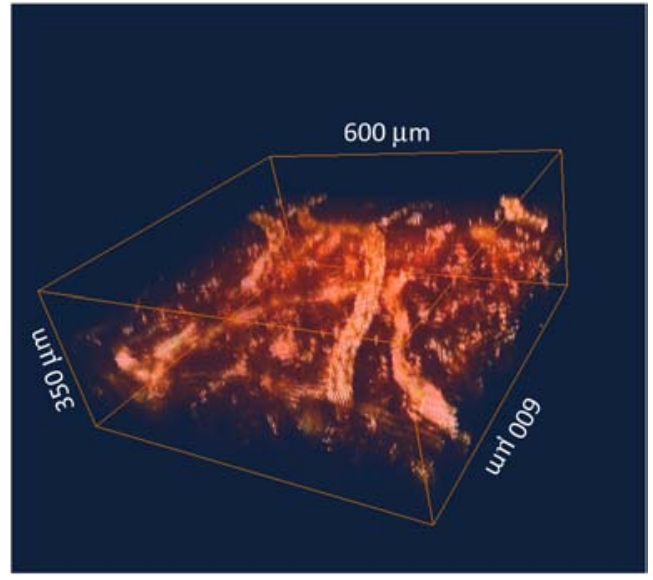

Fig. 4. 3D Reconstruction of the vasculature in the lamina propria of an excised section of mouse colon to a $350 \mu \mathrm{m}$ imaging depth.

Another example is the vasculature under the crypts in the mouse small intestine. Imaging the vasculature network with high resolution is extremely important in biology as all cell behavior is connected to the level of oxygenation of the tissue, and thus to the proximity to blood vessels. Figure 4 shows the 3D reconstruction of the vasculature in the lamina propria of an excised section of mouse colon. The mouse was injected with FITC-Dextran as contrast agent before being euthanized.

The examples of imaging in-depth biological tissue samples by DIVER system show that the applied detection scheme, due to its ability to collect efficiently emission photons, allows imaging of turbid media at depths not accessible by conventional microscopes. This system utilizes a transmission geometry, where excitation is performed from one side of the sample and emission photons collected from its opposite side. In the absence of strong absorption, the multiple scattering in turbid media per $s e$, does not change the detected emission intensity, on the contrary, it increases the number of detected photons. ${ }^{16}$ Using our system we were able to image relatively thick turbid samples (about $1 \mathrm{~cm}$ total thickness), however, the transmission geometry obviously induces some restrictions on the samples that can be imaged by this technique, especially in in vivo imaging experiments. Nevertheless, the method can be useful for in vivo imaging of biological tissue as well and was used for in vivo imaging of intestine and blood vessels where the total thickness of the sample could be in the $1 \mathrm{~cm}$ range. ${ }^{17,19}$ 
For the tissue phantoms samples, which are homogeneous in refraction index, the point spread function (PSF) is not affected by imaging depth and we did not observe any resolution decay with imaging depth. However, it is known that in biological tissue samples, where refraction index varies, the excitation beam wavefront distorts with imaging depth that leads to the loss of resolution in deep layers. This is why tissue phantoms can be imaged deeper than biological tissue samples. Nevertheless, because the DIVER system is mainly designed to collect more efficiently emission photons and it employs a relatively low NA for excitation (NA 0.5), it allows increasing imaging depth in all types of samples when compared to conventional microscopes.

\subsection{SHG images}

SHG is a very powerful imaging modality, often used both ex vivo and in vivo to image biological tissue without the need of exogenous staining. ${ }^{6}$ The detection scheme of the DIVER, due to its transmission geometry, is naturally more suitable and more sensitive for detecting forward propagating SHG signals. Conventional microscopes are bound to collect the back scattered lower intensity portion of SHG photons and also requires a higher excitation laser power for imaging, producing more photodamage to the tissue.

To show the superior performance of the DIVER in acquiring of SHG images, we have imaged several samples that generate SHG signals using both detection schemes at the same time (transmission and epi-geometry). The sensitivity of both detectors was adjusted to give same intensity images for isotropic fluorescence signals.

To compare the epi- and transmission geometry signals we used samples containing dispersed urea crystals, known to give a strong SHG signal. ${ }^{20}$ Figure 5 shows the SHG images of urea crystals dispersed in a scattering silicone resin sample at $1.2 \mathrm{~mm}$ depth. The image acquired by the transPMT is very bright [Fig. 5(a)], while the epi-PMT barely senses any light [Fig. 5(b)] at the same laser power level. The ratio of image intensities was measured to be 50:1. The SHG signal can still be detected by the epi-PMT because the SHG photons are redirected by multiple scattering to the epi-detector.

Collagen, an extremely abundant component of the ECM of biological tissues, gives a strong SHG signal due to a lack of a center of symmetry in its

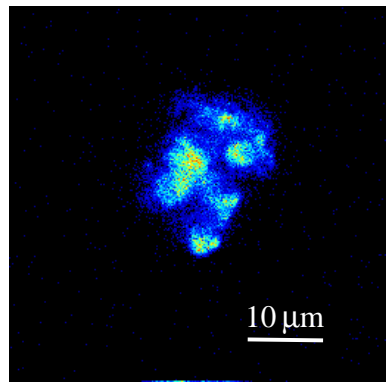

(a)

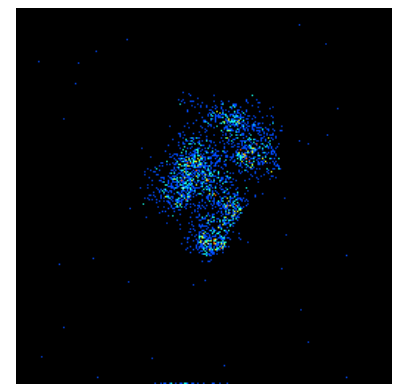

(b)
Fig. 5. SHG images of urea crystals dispersed in a scattering silicone resin sample at $1.2 \mathrm{~mm}$ depth acquired by the transPMT (a) and epi-PMT (b).

structure. Figure 6 shows the images of collagen fibers in thick collagen matrix acquired by both PMTs. The image acquired by the trans-PMT presents a higher intensity than the one acquired by the epi-PMT. The ratio of the intensities of these images was measured to be $\sim 250: 1$. This ratio significantly increases with imaging depth, as shown in Fig. 7, which makes the DIVER's superior detection

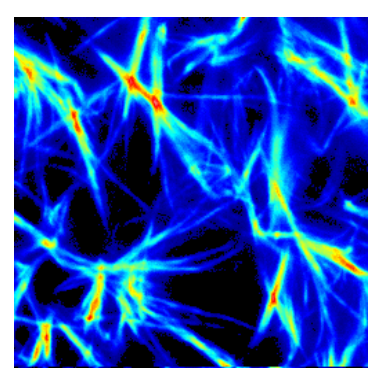

(a)

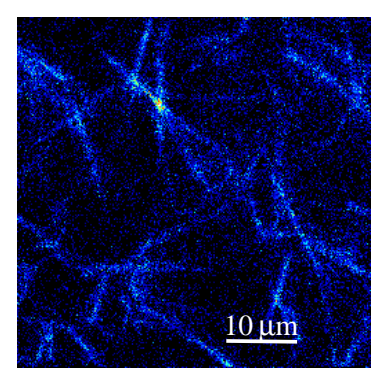

(b)
Fig. 6. SHG images of thick collagen matrix acquired by the trans-PMT (a) and epi-PMT (b).

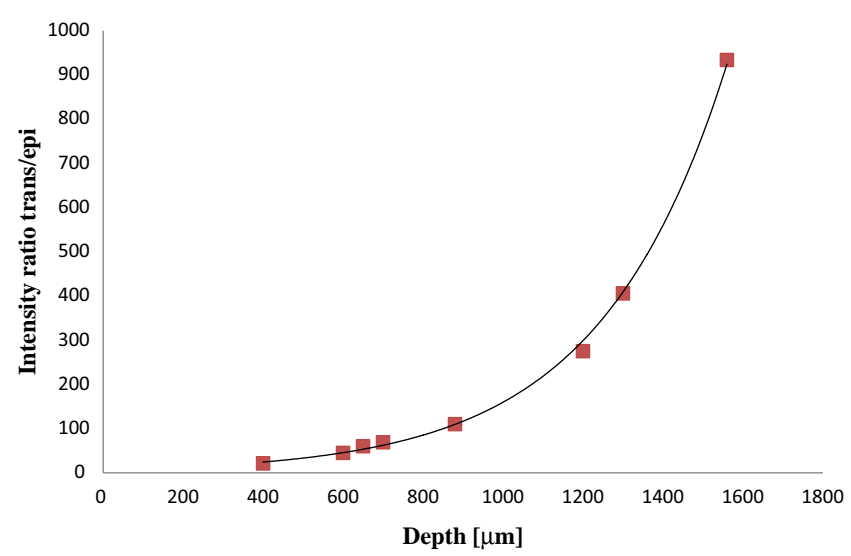

Fig. 7. Plot of ratio of SHG image intensities acquired by the trans-PMT and epi-PMT as a function of imaging depth. 


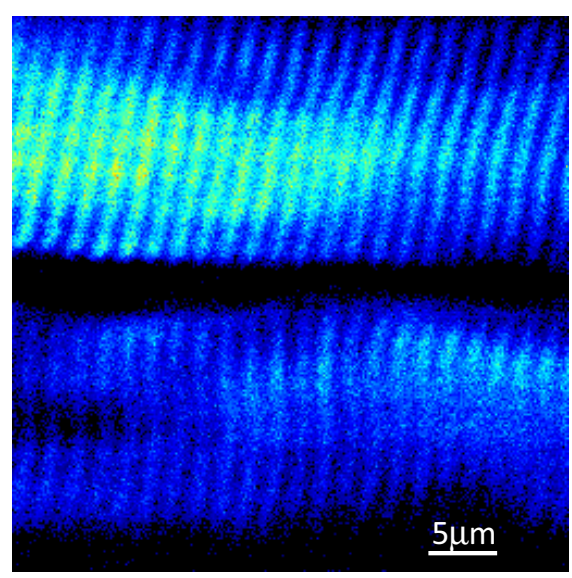

Fig. 8. SHG image of muscle fibers in the mouse medial thigh muscle acquired with the DIVER's trans-detector.

method even more suitable for SHG imaging in deeper layers.

Myosin is another strong SHG signal generator widely present in biological tissue, such as muscles. Figure 8 shows the image of muscle fibers in sarcomeres of the mouse medial thigh muscle acquired with the DIVER's trans-detector, which were imaged to the depths of about $600 \mu \mathrm{m}$, not accessible by conventional microscopes.

\subsection{FLIM}

FLIM uses fluorophore lifetime (fluorescence decay) to generate images rather than the intensity or abundance of the fluorophore itself. Since each fluorophore has a specific signature lifetime, FLIM imaging can be used to identify different fluorophores with overlapping spectra, a task that cannot be achieved with the traditional, steady-state fluorescence microscopy. ${ }^{21}$ The FLIM capability of the DIVER adds a crucial contrast mechanism. Tissues that appear homogeneous on the basis of fluorescence intensity could have very different spatial distribution of lifetime.

The DIVER is capable of acquiring FLIM images at higher depths than conventional microscopes. FLIM data acquired at such depths have not been previously presented to the best of our knowledge. FLIM images of fluorescent beads of different color and size dispersed in a scattering silicone tissue phantom with different dimensions were imaged using the DIVER (Fig. 9). In Fig. 9(a), we show a $10-\mu \mathrm{m}$ red bead at the depth of $1.2 \mathrm{~mm}$, while Fig. 9(b) shows a $15-\mu \mathrm{m}$ yellow-green fluorescent bead at the depth of $3 \mathrm{~mm}$. In Fig. 9(c), the FLIM phasor analysis shows the different beads highlighted according to their lifetimes, each having distinct locations on the phasor.

Figure 10 shows an intensity image of the crypts (a), the FLIM images (b), and the phasor-plot (c) of the mouse small intestine obtained at $740 \mathrm{~nm}$ which measure tissue intrinsic autofluorescence. The intensity image does not provide very good contrast, due to the weak autofluorescence of the tissues in the crypt. However, the lifetime is independent of fluorophore concentration and in the FLIM images the crypts (NADH) are clearly discerned along with other tissue types. The green area represents the NADH content in the crypts and at the top of the villi, while the yellow one identifies the muscle layer

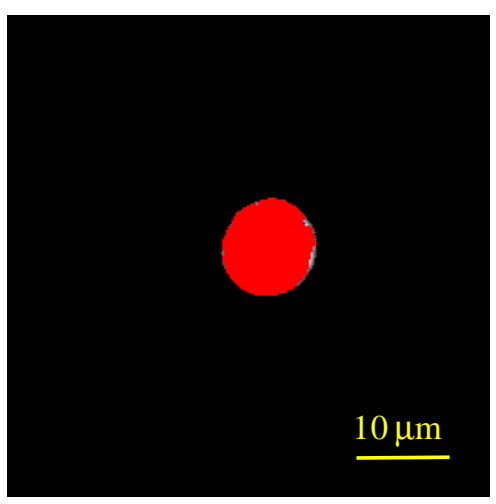

(a)

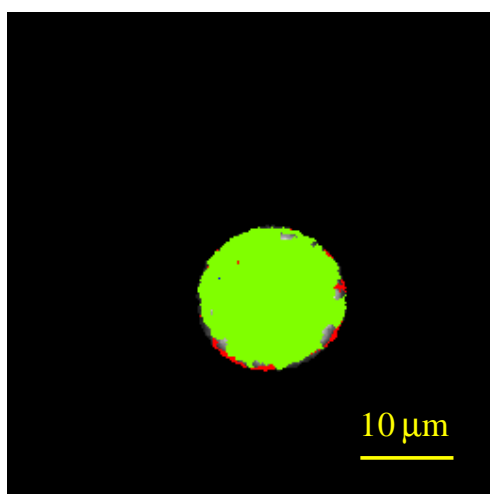

(b)

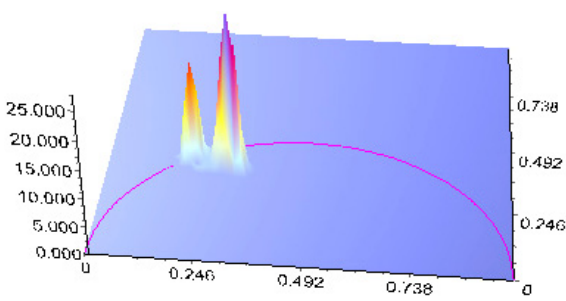

(c)

Fig. 9. FLIM images of fluorescent beads of different colors and sizes dispersed in a scattering silicone tissue phantom. FLIM image of a $10 \mu \mathrm{m}$ red bead acquired at the depth of $1.2 \mathrm{~mm}$ (a). FLIM image of a $15 \mu \mathrm{m}$ yellow-green fluorescent bead acquired at the depth of $3 \mathrm{~mm}$ (b). Phasor-plot (c). 


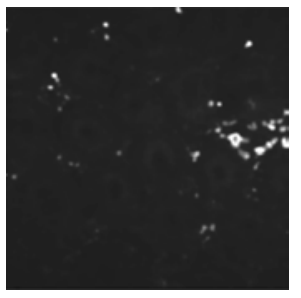

(a)

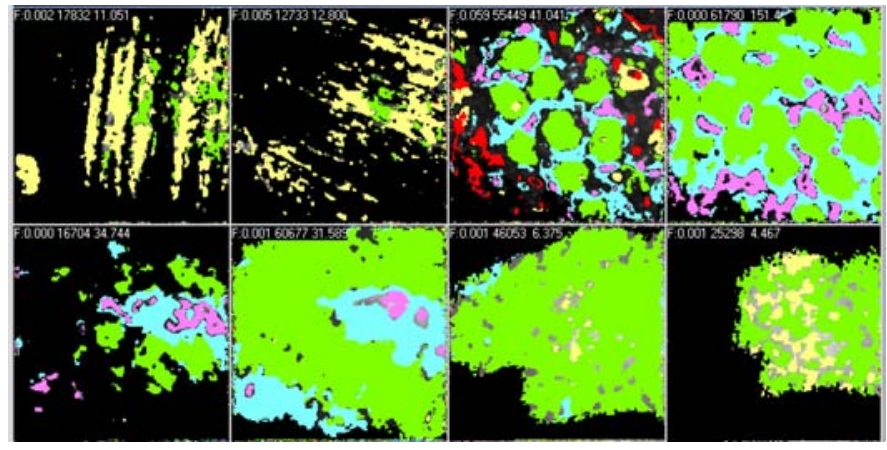

(b)

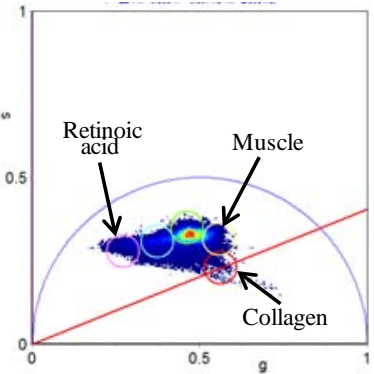

(c)

Fig. 10. Intensity image of the crypts (a), the FLIM images (b), and the phasor-plot (c) of the mouse small intestine.

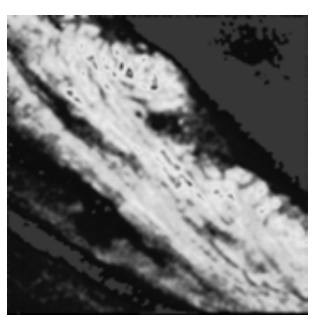

(a)

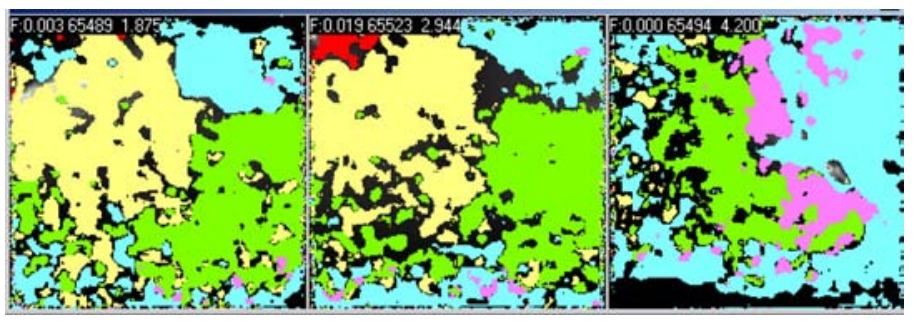

(b)

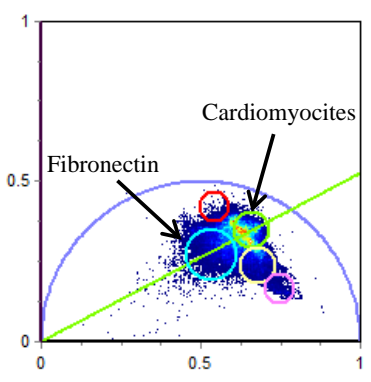

(c)

Fig. 11. Intensity image (a), FLIM images (b) and the phasor-plot (c) of the mouse heart. The image was acquired to $700 \mu \mathrm{m}$ depth.

under the lamina propria. The pink area corresponds to retinoic acid, while the red area corresponds to collagen.

Figure 11 shows an intensity image (a), FLIM images (b) and the phasor-plot (b) of the mouse heart. While the intensity image only provides rough structural information with the FLIM image, different cells and tissue types can be clearly discerned. The green region represents the cardiomyocites, while the blue one is fibronectin.

The reason we can differentiate different ex vivo tissue types using phasor-plot is most likely due to specific autofluorescence originating in each organ and how the NADH is distributed in these tissue and its association with different tissue specific proteins.

\section{Conclusion}

In this paper, we have introduced the DIVER, a two-photon fluorescence scanning microscope. The system is capable of acquiring fluorescence, SHG, and fluorescence lifetime-based images in turbid biological and nonbiological samples, up to the depth greater than $4 \mathrm{~mm}$ with micron resolution by virtue of an innovative ultra-sensitive detection system. The specially designed detector collects emission photons from a wide area of the sample with high efficiency. The advantage of the system is due to the full index matching of the optical path between the sample and the detector window, loss of photons due to reflections at the boundaries are greatly diminished making the DIVER highly sensitive to low-level signals unlike any other system presently available. These features allow the system to image roughly eight times deeper in turbid media than conventional two-photon fluorescence microscopes.

Since SHG signals intrinsically tend to propagate in the direction of the excitation light, the transmission geometry of the DIVER's detection system gives it the upper hand on conventional microscopes in SHG imaging. In-depth FLIM imaging capabilities have also been demonstrated with an efficient lifetime-based separation up to the depth of $3 \mathrm{~mm}$. 


\section{Acknowledgments}

Funding was provided by National Institutes of Health P50 GM076516, by the National Center for Research Resources (5P41RR003155-27) and the National Institute of General Medical Sciences (8 P41 GM103540-27). The authors would like to thank, Dr. Chi-Li Chiu, Dr. R. Saager, Dr. M. Waterman, and Dr. Robert Edwards for providing us with the samples.

\section{References}

1. D. O'Malley, "Imaging in depth: Controversies and opportunities," Methods Cell Biol. 89, Ch. 5, 95-128 (2008).

2. P. Theer, M. T. Hasan, W. Denk, "Two-photon imaging to a depth of 1000 micron in living brains by use of a Ti:Al2O3 regenerative amplifier," Opt. Lett. 28(12), 1022-1024 (2003).

3. D. Kobat, M. E. Durst, N. Nishimura, A. W. Wong, C. B. Schaffer, C. Xu, "Deep tissue multiphoton microscopy using longer wavelength excitation," Opt. Express 17, 13354-13364 (2009).

4. R. Cicchi, D. D. Sampson, D. Massi, F. S. Pavone, "Contrast and depth enhancement in two-photon microscopy of human skin ex vivo by use of optical clearing agents," Opt. Express 13, 2337-2344 (2005).

5. C. A. Combs, A. V. Smirnov, J. D. Riley, A. H. Gandjbakhche, J. R. Knutson, R. S. Balaban, "Optimization of multiphoton excitation microscopy by total emission detection using a parabolic light reflector," J. Microsc. 228, 330-337 (2007).

6. P. J. Campagnola, C. Dong, "Second harmonic generation microscopy: Principles and applications to disease diagnosis," Laser Photon. Rev. 5(1), 1326 (2011).

7. W. R. Zipfel, R. M. Williams, W. W. Watt, "Nonlinear magic: Multiphoton microscopy in the biosciences," Nat. Biotechnol. 21(11), 1369-1377 (2003).

8. X. Chen, C. Raggio, P. J. Campagnola, "Secondharmonic generation circular dichroism studies of osteogenesis imperfecta," Opt Lett. 37, 18 (2012).

9. O. Nadiarnykh, R. B. LaComb, M. A. Brewer, P. J. Campagnola, "Alterations of the extracellular matrix in ovarian cancer studied by second harmonic generation imaging microscopy," BMC Cancer 10, $94(2010)$.

10. V. Ajeti, O. Nadiarnykh, S. M. Ponik, P. J. Keely, K. W. Eliceiri, P. J. Campagnola, "Structural changes in mixed $\mathrm{Col} \mathrm{I/Col} \mathrm{V} \mathrm{collagen} \mathrm{gels} \mathrm{probed}$ by SHG microscopy: Implications for probing stromal alterations in human breast cancer," Biomed. Opt. Express 2(8), 2307-2316 (2011).

11. S. V. Plotnikov, A. M. Kenny, S. J. Walsh, B. Zubrowski, C. Joseph, V. L. Scranton, G. A. Kuchel, D. Dauser, M. Xu, C. C. Pilbeam, D. J. Adams, R. P. Dougherty, P. J. Campagnola, W. A. Mohler, "Measurement of muscle disease by quantitative second-harmonic generation imaging," J. Biomed. Opt. 13(4) (2008).

12. H. C. Ishikawa-Ankerhold, R. Ankerhold, G. P. C. Drummen, "Advanced fluorescence microscopy techniques - FRAP, FLIP, FLAP, FRET and FLIM," Molecules 17, 4047-4132 (2012).

13. P. I. Bastiaens, A. Squire, "Fluorescence lifetime imaging microscopy: Spatial resolution of biochemical processes in the cell," Trends Cell Biol. 9, 48-52 (1999).

14. E. B. van Munster, T. W. Gadella, Jr., "Suppression of photobleaching-induced artifacts infrequency-domain FLIM by permutation of the recording order," Cytometry A 58, 185-194 (2004).

15. M. A. Digman, V. R. Caiolfa, M. Zamai, E. Gratton, "The phasor approach to fluorescence lifetime imaging analysis," Biophys J. 94(2), L14-L16 (2008).

16. V. Crosignani, A. S. Dvornikov, E. Gratton, "Enhancement of imaging depth in turbid media using a wide area detector," J. Biophotonics 4(9), 592-599 (2011).

17. V. Crosignani, A. S. Dvornikov, J. S. Aguilar, C. Stringari, R. Edwards, W. W. Mantulin, E. Gratton, "Deep tissue fluorescence imaging and in vivo biological applications," J. Biomed. Opt. 17(11), 116023-1-7 (2012).

18. F. Ayers, A. Grant, D. Kuo, D. J. Cuccia, A. J. Durkin, "Fabrication and characterization of silicone-based tissue phantoms with tunable optical properties in the visible and near infrared domain," Proc. SPIE 6870, 687007-1-9 (2008).

19. V. Crosignani, A. Dvornikov, E. Gratton "Ultradeep imaging of turbid samples by enhanced photon harvesting," Proc. SPIE 8588, 858810-858811 (2013).

20. S. Romani, C. Razzetti, M. Zha, C. Paorici, "Evaluation of the effective second harmonic generation coefficient of monomethyl urea single crystals," Phys. Status Solidi (B) 197, 271 (1996).

21. C. Chang, D. Sud, M. Mycek, "Fluorescence lifetime imaging microscopy," Methods Cell Biol. 81, 495524 (2007). 